Acoustic myography is the recording of sounds produced by contracting muscle. These sounds become louder with increasing force of contraction. We have compared muscle sounds with surface EMG to monitor the dissociation of electrical from mechanical events (presumably, the loss of excitation-contraction coupling) which occur with motor unit fatigue. Acoustic signals were amplified using a standard phonocardiograph, recorded on FM magnetic tape, and digitally analyzed. Muscles were examined at rest, with intermittent contractions, and with sustained contractions. We found that with fatigue, the acoustic amplitude decayed, but the surface EMG amplitude did not. With decreased effort, however, the acoustic and the surface EMG amplitudes declined simultaneously. By simultaneously recording acoustic signals and needle EMG, individual motor units were resolved acoustically in two muscles with decreased numbers of motor units and increased motor unit size. Fasciculations also produced acoustic signals, although no acoustic signal has yet been found that correlates with fibrillations. Analysis of acoustic signals from muscle provides a noninvasive method for monitoring motor unit fatigue in vivo. It may also be useful in distinguishing muscle fatigue from decreased volition.

MUSCLE \& NERVE 8:189-194 1985

\title{
ACOUSTIC MYOGRAPHY: A NONINVASIVE MONITOR OF MOTOR UNIT FATIGUE
}

\author{
DANIEL T. BARRY, MD, PhD, STEVEN R. GEIRINGER, MD, \\ and RICHARD D. BALL, MD, PhD
}

Skeletal muscle generates sounds when contracting. These sounds are audible, for example, by pressing the palms against one's ears with the fingers pointing up, and they resemble the rumble of heavy rain on a roof. Previous investigators recorded these continuous, low frequency sounds and demonstrated that they are not qualitatively altered by temperature variation or momentary cessation of blood flow. ${ }^{10,12,13,17}$ Oster and Jaffee ${ }^{13}$ found a linear relationship between the root mean squared (RMS) amplitude and the force of contrac-

From the Department of Physical Medicine and Rehabilitation. University of Michigan. Ann Arbor, M!.

Acknowledgments: We wish to thank Dr. Gerald Oster for many helpful suggestions and discussions, and for a critical reading of an earlier version of this paper. We thank Dr. Andrew Buda for the use of the irex phonocardiograph and microphone. We thank Dr. Kathryn A. Stolp-Smith for assistance with data collection and analysis. We thank Ms. Nancy Asin for editorial assistance. Parts of this paper were presented at the 1984 AAEE Meetings in Kansas City, MO, as the winner of the 1984 Young Investigator Award

Address reprint requests to Dr. Barry at the University of Michigan, De partment of Physical Medicine and Rehabilitation, E3129 University Hospital. Box 33, Ann Arbor, Mł 48109-0010.

Received for publication May 1, 1984; revised manuscript accepted for publication September 21, 1984

$0148-639 \times / 0803 / 0189 \$ 04,00$

c) 1985 John Wiley \& Sons, inc.

tion of wrist flexor forearm muscles. They calculated autocorrelation functions and noted that the power spectral density function of muscle sounds reaches a maximum at approximately 25 $\mathrm{Hz}$, whereas there is only a small percentage of the total power at frequencies greater than $100 \mathrm{H}$.

Occasionally, discrete acoustic spikes are resolved. Gordon and Holbourn ${ }^{8}$ reported spikes in recordings from orbicularis oculi and correlated them with individual motor units seen with surface electromyography. The spikes were usually diphasic, with durations of $5-15 \mathrm{msec}$, and the sound signal followed the surface electrical signal by $0.4-2.0 \mathrm{msec}$. They were not, however, able to resolve acoustic spikes from muscles in the trunk or extremities.

Brozovich and Pollack ${ }^{3,4}$ reported that acoustic spikes of shorter duration $(0.4 \mathrm{msec})$ occur after maximal stimulation of frog sartorius muscle in vitro. These spikes are not related to motor unit activity and may represent fundamental units which are summed to produce the spikes and continuous sounds recorded in vivo.

We hypothesize that if the acoustic signal is an intrinsic property of contraction, unaffected by electrical activity of the muscle, then we should be able to monitor the dissociation of electrical from 
mechanical events (presumably, the loss of excitation-contraction coupling) in a muscle as it fatigues. The total spectral power of the surface EMG does not correlate well with force production in sustained, submaximal isometric contractions of constant force. In fact, the total spectral power (or RMS amplitude) of the surface EMG progressively increases during sustained constant force submaximal contractions and may remain elevated while force is decreasing significantly. ${ }^{5,6,11,14}$ Because the surface EMG signal reflects muscle fiber action potentials and the acoustic signal theoretically reflects muscle fiber contraction, the loss of excitationcontraction coupling should result in a decrease in the acoustic signal relative to the surface EMG.

\section{MATERIALS AND METHODS}

Sounds were detected with a standard phonocardiograph (Irex 152-103HSP-1 heart sound/pulse module and heart sound microphone piece/N12010120-0131D0002; Irex Medical Systems, Upper Saddle River, NJ) and recorded on magnetic tape with an HP3960 series FM recorder (HewlettPackard, Mountain View, CA). These signals were sampled at $1-\mathrm{msec}$ intervals and digitally analyzed after 12 bit A/D conversion. The graphic display reduces the data range to $0-511$ when producing plots.

The frequency responses of the microphone, phonocardiograph, and tape recorder were measured and the system was found to have a band width of $20-1500 \mathrm{~Hz}$ within $\pm 3 \mathrm{~dB}$. Below $20 \mathrm{~Hz}$, the response decayed by $6 \mathrm{~dB}$ per decade. The microphone was calibrated for linearity to variation in acoustic pressures using a GR 1988 precision integrating sound level meter (GenRad, Concord, MA) and was found to have linear characteristics. Air pressure levels of $90 \mathrm{~dB}$ produced a $50-\mathrm{mV}$ transducer output at $60 \mathrm{~Hz}$, which corresponded to a $1.5-\mathrm{V}$ output from the phonocardiograph (gain $=30$ ). These levels are not directly translatable into intensity of muscle sounds at the skin surface, because the suction cup microphone creates an acoustic cavity and acoustic reflection occurs at the skin-air interface.

Acoustic myography (AMG) and surface EMG signals were simultaneously recorded from the biceps brachii of normal adult volunteers during isometric contractions. In the first set of experiments, data were collected according to the following protocol. Five subjects were sitting with the forearm parallel to the ground; weights of $0,5,10$, $12.5,15$, and $20 \mathrm{lb}$ were held in the palm. Each weight was held for 20 seconds with 10 -second rests between weights. The entire cycle was repeated five times, with 15 seconds between sets. After digitization, RMS amplitudes were calculated and normalized to a percentage of mean value for each individual. These data were combined to produce overall mean values at each weight for each cycle. The data from all five cycles were combined to produce mean values at each weight for the entire experiment. Standard deviations of these normalized values among the five subjects were calculated. In the second experiment, 10 volunteers were studied ( 6 unilaterally and 4 bilaterally- 14 muscles total) while they sustained isometric contraction with the elbow flexed to $90^{\circ}$ and the forearm strapped into a posterior shell. Volunteers initially exerted a $75 \%$ maximal voluntary contraction (MVC), which was maintained as long as possible; as force production decreased, data continued to be recorded until force dropped to $35 \%$ of the original MVC. Visual feedback of force production was provided.

Data were divided into 20 sections, each comprising $5 \%$ of the total trial time for each individual. Again, RMS amplitudes were calculated and normalized to a percentage of the mean values for force, $A M G$, and EMG signals of each trial. These data from all 14 trials were combined to produce an overall mean value for each of the 20 sections. Least mean squared linear fitting, with Student's $t$ test used to define $95 \%$ confidence limits, was then done to determine the relationship of $A M G$ to force and EMG to force.

Variations of absolute AMG amplitudes among individuals were not addressed in this study, because the use of a large (approximately 2 -in. diameter) air-coupled microphone makes identical coupling to each individual difficult. Future studies will incorporate an electret microphone ${ }^{16}$ of approximately 0.5 -in. diameter, with a unity impedance match to skin.

AMG and needle EMG were recorded simultaneously from two patients with muscles having large motor units secondary to denervation and subsequent axonal sprouting (femoral nerve injury 6 months previously and ulnar nerve lesion 5 months prior to testing). Recordings were also made with needle EMG and acoustic pick-up alone to assure that the signals were not affected by cross-talk between the two modalities. The microphone was electrically isolated from the skin with a porous cloth to demonstrate that the acoustic data are not fundamentally changed by losing contact with the skin. Arrival times of EMG and AMG signals were calibrated by simulta- 
neously producing electrical and acoustic signals and measuring the arrival times through the instrumentation.

\section{RESULTS}

The acoustic signal RMS amplitude increases with increasing force of contraction (Fig. 1, A and B). The dynamic range of this signal from biceps brachii with the arm at rest versus supporting a 20 lb load is approximately 25 . The relationship of RMS amplitude to load is approximately linear in the mid-range, with nonlinearities appearing in both low load and high load conditions. Isometric weight lifting with repeated sets of $0,5,10,15$, and $20 \mathrm{lb}$ weights produces increasing acoustic signal amplitudes with successive sets at high

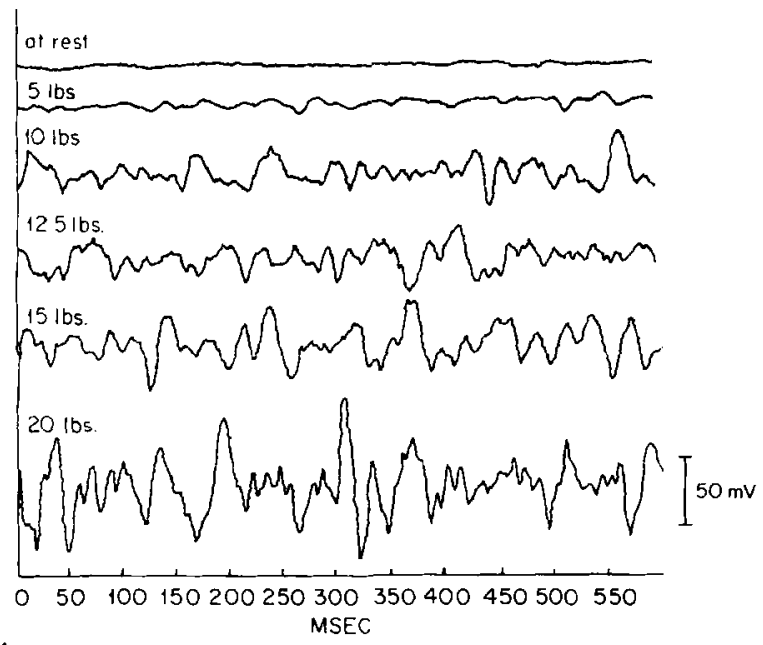

A

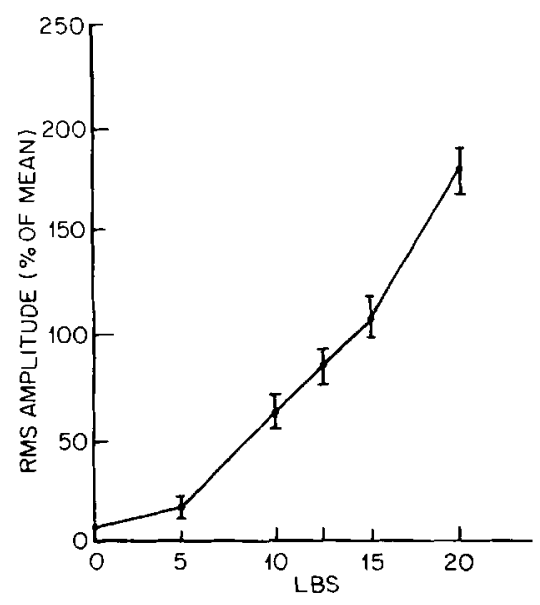

FIGURE 1. (A) Examples of short segments of acoustic data from biceps brachii of a normal adult volunteer with different weights placed in the palm. (B) Plot of acoustic amplitude versus load, averaged over all five cycles and all five subjects. Error bars denote standard deviations of normalized values among the five subjects.

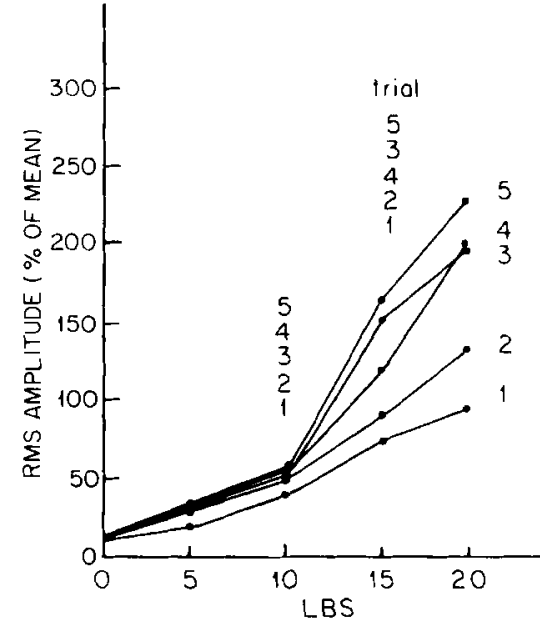

FIGURE 2. Successive trials of holding weights produces increasing RMS amplitudes of the acoustic signal.

weights (Fig. 2). The amplitudes at low weight do not change with successive sets.

Isometric exercise with maximal effort produces an AMG signal that parallels declining force with fatigue. Figures 3 and 4 demonstrate the relationship of AMG to force and EMG to force during sustained maximal effort as force drops from $75 \%$ MVC to $35 \%$ MVC. Linear least squares fitting reveals the parameters shown in Table 1. The AMG signal follows the decline in force throughout the experiment, whereas the FMG signal decays only below 45\% MVC (approximately $75 \%$ of mean force). When two of the volunteers were told to reduce effort during isometric exercise, the $A M G$, surface FMG, and force levels all decreased simultaneously (Fig. 5).

Simultaneous recording of AMG and needle EMG signals occasionally reveals a motor unit which appears in both tracings (Fig. 6). These have been seen in limb muscles of two patients who had large motor unit action potentials and decreased numbers of motor units shown by needle EMG. They have not yet been demonstrated in nomal limb muscles. The microphone must be placed directly over the needle, the subject must be able to activate only a few motor units at one time, and the positions of the needle and microphone must be systematically varied until simultaneous signals appear. Once located, a motor unit is reproducible and reliably appears in both signals. Motor units recorded from vastus lateralis and first dorsal interosseus had EMG durations of approximately 10 msec, with AMG durations of $40 \mathrm{msec}$. The waveform of the $A M G$ signal tended to be triphasic or polyphasic. Acoustic signals correlated 


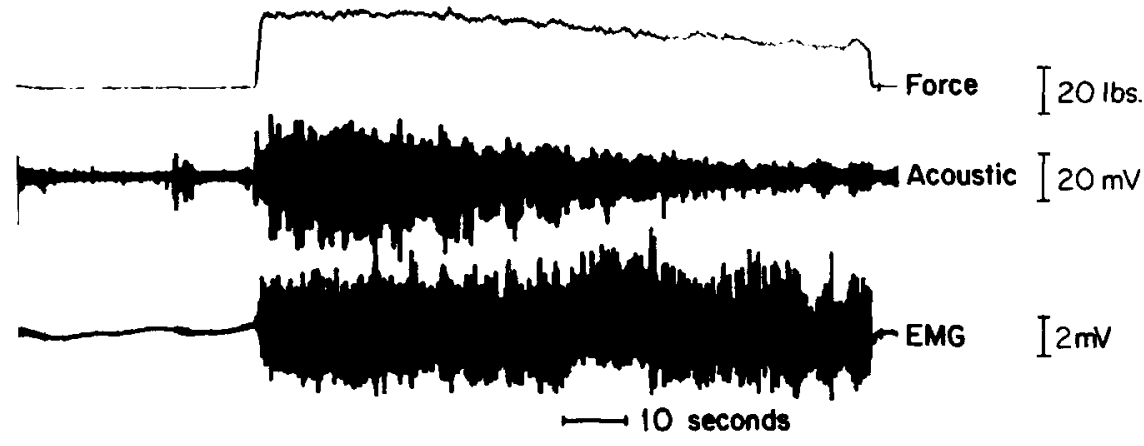

FIGURE 3. An example of simultaneous recording of AMG, surface EMG, and force with high initial force $(75 \%$ $\mathrm{MVC}$ ) held as long as possible. with visible fasciculations were seen in both normal and partially denervated muscle (Fig. 7). We have not yet identified an acoustic correlate to fibrillation.

\section{DIScussion}

The data presented in this article support the hypothesis that the RMS amplitude of the acoustic signal produced during skeletal muscle contraction is highly correlated with force production. Because the RMS amplitude of the surface EMG provides an index of muscle fiber electrical activity, comparison of acoustic and electrical data provides a means of monitoring the dissociation of electrical and mechanical events that frequently occurs with fatigue. Below $45 \%$ of MVC, the surface EMG signal started to decay. Decreased subject motivation may be a factor in this decay, as volunteers frequently stated that the experiment was exhausting, became painful toward the end, and that observing a declining force value was discouraging. We will evaluate this possibility first by providing visual feedback of the surface EMG signal amplitude as a "measure of effort" rather than force production. Second, we will externally stimulate the motor nerve supramaximally at periodic intervals during the experiment. If the muscle is being fully exerted voluntarily, there will be no additional increase in force production with the neural stimulus. ${ }^{2} \mathrm{Al}$ though decreased motivation may be the cause of the EMG signal decay, late failure at the neuromuscular junction cannot be ruled out.

Although the RMS amplitude of the acoustic signal is highly correlated with force production, the exact relationship depends on several factors. When the acoustic signal is monitored under conditions where fatigue develops rapidly (e.g., 1-2 minutes of isometric contraction starting at $75 \%$ of MVC), the decay of the acoustic signal correlates well with the loss of force production. However, when the acoustic signal is measured over relatively long periods of time with periodic rests, the acoustic signal increases with time. There are several possible explanations for the increase in the acoustic signal under these conditions, including: (1) increased efficiency of acoustic transmission as muscle swells secondary to osmotic phenomena during prolonged exercise; (2) increased physio-
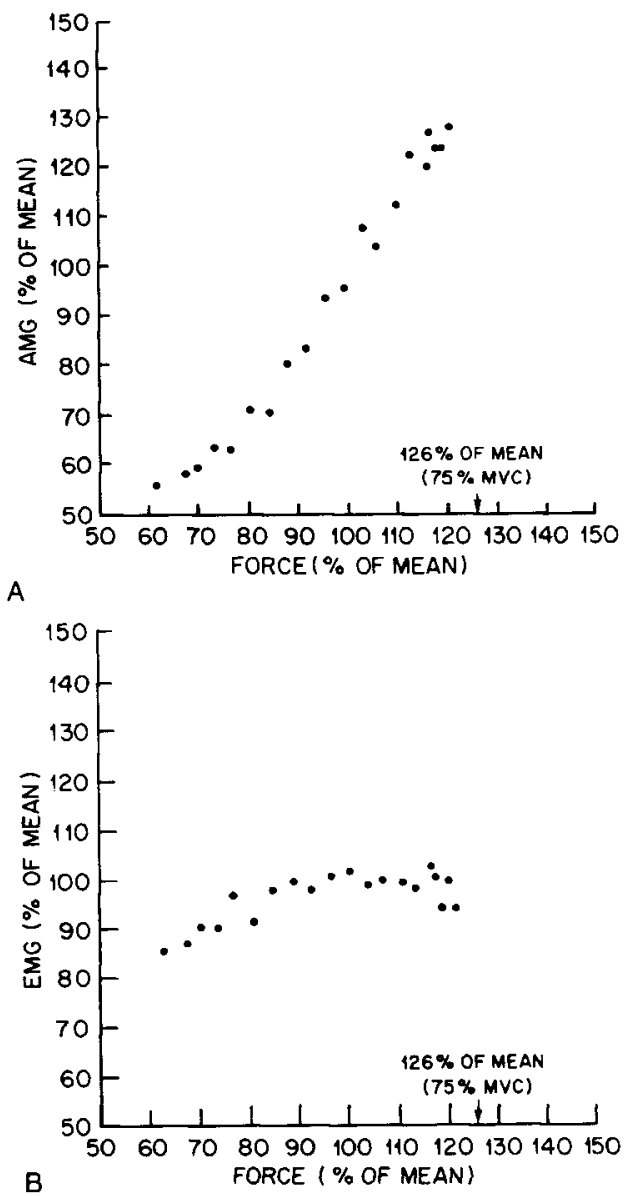

FIGURE 4. (A) AMG versus force-averaged results from 14 trials. Force decays from $75 \%$ MVC to $35 \%$ MVC. Each point represents $5 \%$ of the trial time. Note that the points with higher force generally correspond to the earlier part of the experiment; as time progresses, force decreases, moving from right to left. (B) Surface EMG versus force. 


\begin{tabular}{|c|c|c|c|c|}
\hline & $A M G$ & $\begin{array}{c}95 \% \\
\text { Confidence }\end{array}$ & EMG & $\begin{array}{c}95 \% \\
\text { Confidence }\end{array}$ \\
\hline \multicolumn{5}{|l|}{ All 20 points } \\
\hline Slope & 1.38 & \pm 0.11 & 0.182 & \pm 0.10 \\
\hline Intercept & -0.38 & \pm 0.11 & 0.82 & $=0.10$ \\
\hline \multicolumn{5}{|c|}{14 Points with force $>75 \%$ of mean } \\
\hline Slope & 1.56 & \pm 0.15 & -0.036 & \pm 0.12 \\
\hline Interceot & -0.59 & \pm 0.17 & 0.92 & \pm 0.12 \\
\hline
\end{tabular}

Linear least mean squared fitting to the data in Fig. 4, using force as the independent variable, with AMG ano EMG amplitudes as the dependent variatiles. The EMG signa/ shows no decay when the data are truncated below $45 \%$ MVC $(75 \%$ mean force)

logic tremor with prolonged exercise; or $(3)$ some undefined phenomenon related to motor unit recruitment. We are curently examining each of these possibilities.

The above data suggest that $A M G$ may be a useful technique to noninvasively monitor fatigue. Most current noninvasive techniques for monitoring muscular fatigue are based on spectral compression of the surface EMG, ${ }^{6,11,14,15}$ which requires exacting instrumentation and is nontrivial to perform in real time. It is well known that the RMS amplitude of the surface EMG does not correlate well with fatigue. ${ }^{2,6,7}$ Monitoring RMS AMG signals therefore provides a technique which is relatively simple, does not require sophisticated equipment, and can easily be done in real time. Monitoring fatigue by comparing the RMS am-

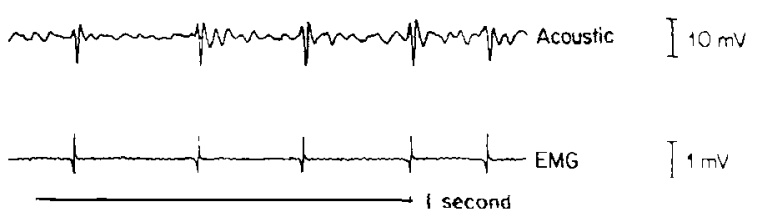

FIGURE 6. Simultaneous recordings of AMG and needle EMG from the vastus lateralis of a subject who sustained a traumatic femoral nerve lesion 6 months previously. Individual motor units are resolved acoustically.

plitudes of AMG and surface EMG may also be useful in avoiding the "overwork" phenomenon during therapeutic exercise, ${ }^{1,9}$ as fatigue appears to be correlated with a decrease in the ratios of RMS AMG to EMG signals. Comparison of AMG and EMG amplitudes also provides an indicator of volition (Fig. 5): when a subject chooses to reduce ef-

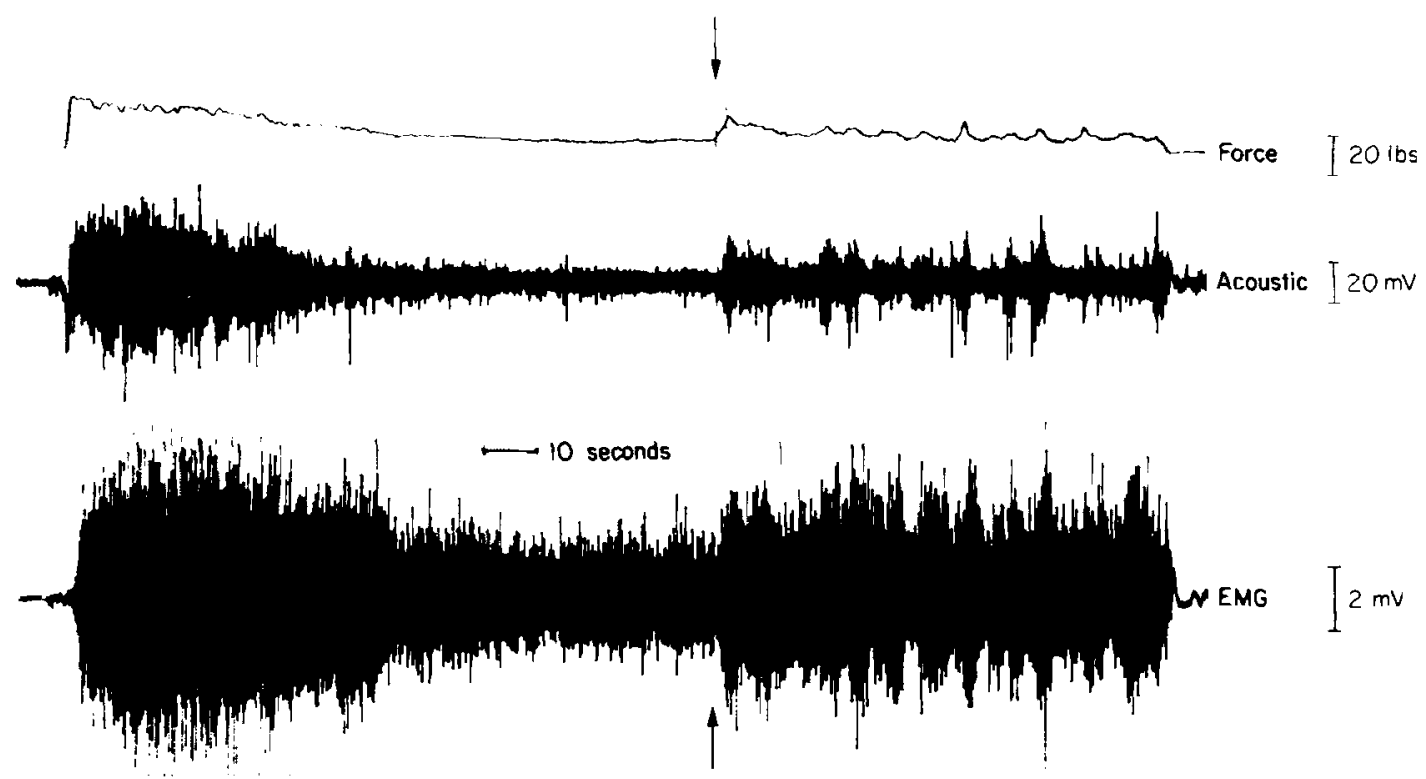

FIGURE 5. When force declines due to decreased volition rather than fatigue, the AMG amplitude, surface EMG amplitude, and force all decay simultaneously. An increase in effort (arrow) produces a simultaneous increase in all three signal amplitudes. Note how closely the AMG signal amplitude follows small changes in force exerted. 


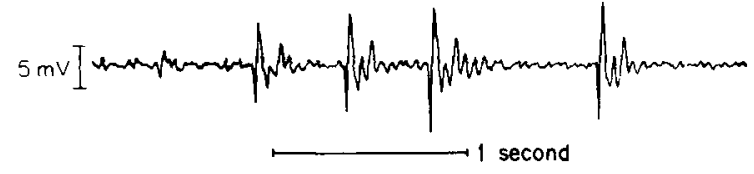

FIGURE 7. These acoustic signals were recorded from a muscle (vastus medialis) which was observed to be fasciculating The acoustic spikes were visually correlated with the fasciculations.

fort, the EMG, AMG, and force amplitudes decline simultaneously. This could be a valuable parameter in distinguishing motor unit fatigue from lack of effort

The detection of individual acoustic spikes that correlate with needle EMG confirms that individual motor units can be resolved acoustically. The extended duration of the acoustic motor unit signal compared to the needle EMG waveform makes AMG more susceptible to interference due to overlap of multiple motor unit signals. We have been able to resolve motor units in limbs of two patients with decreased numbers of motor units and increased motor unit action potential size.
An explanation for the sound, first proposed by Gordon and Holbourn, ${ }^{8}$ is that a pressure wave is produced by a traveling wave contraction along muscle fibers. As the fiber contracts axially, it also expands radially, initiating a pressure wave within the tissue. This mechanism would predict that the waveform of an acoustic spike should be monophasic or diphasic. The existence of polyphasic spikes may be due to interference among several fibers within a motor unit. This would be produced by variations in distance from the microphone and in contraction velocity among different fibers. Analysis of the acoustic waveform parameters, such as amplitude, duration, and configuration, may have diagnostic or prognostic implications, much as they do in EMG.

In summary, $\mathrm{AMG}$ represents a new modality for analyzing muscular activity. As a direct indicator of contraction, AMG allows in vivo measurement of the dissociation of electrical and mechanical events in muscle with fatigue. Because resolution of individual motor units has been demonstrated, it is possible that waveform analysis will reveal prognostic or diagnostic information.

\section{REFERENCES}

1. Bennett RL. Knowlton GC: Overwork weakness in partially denervated skeletal muscle. Clin Orthop 12:22-29, 1958.

2. Bigland-Ritchie B: EMG and fatigue of human voluntary and stimulated contractions, in Porter R, Whelan J (eds): Human Muscle Fatigue and Physiological Mechanisms (Ciba Foundation Symposium 82). London, Pitman Medical, 1981, pp $130-156$.

3. Brozovich FV, Pollack GH: Muscle contraction generates discrete sound bursts. Biophys / 41:35-40, 1983 .

4. Brozovich FV, Pollack GH: Muscle sounds: Observations with improved transducers. Biophys $/ 45: 106 a, 1984$.

5. Chaffin DB, Lee M, Freivalds A: Muscle strength assessment from EMG analysis. Med Sci Sports Exerc 12:205-211, 1980.

6. DeLuca CJ: Physiology and mathematics of myoelectric signals. IEEE Trans Biomed Eng 26:313-325, 1979.

7. Edwards RG, Lippold OCJ: The relation between force and integrated electrical activity in fatigued muscle. $J$ Physiol (lond) 132:677-681, 1956

8. Gordon G, Holbourn AHS: The sounds from simple motor units in a contracting nuscle. J Physiol (Lond) 107:456-464, 1948

9. Herbison GJ, Jaweed MM, Ditunno JF: Reinnervating rat skeletal muscle: Effect of $35 \%$ grade treadmill exercise. Arch Phys Med Rehabil 63:313-316, 1982.

10. Herroun EF, Yeo GF: Note on the sound accompanying the single contraction of skeletal muscle. I Physiol (Lond) 6:287-292, 1885

11. Maron B: Human motor unit activity during the onset of muscle fatigue in submaximal isometric isotonic contraction. EurJ Appl Physiol 46:271-281, 1981.

12. Oster G: Muscle sounds. Sci Am 250:108-115, 1984.

13. Oster $G$, Jaffe JS: Low frequency sounds from sustained contraction of human skeletal muscle. Biophys $f$ 30:119127,1980 .

14. Sadoyama T, Miyano H: Frequency analysis of surface EMG applied to evaluation of muscle fatigue. Eur / Appl Physiol 47:239-246, 1981.

15. Stulen FB, DeLuca CJ: Muscle fatigue monitor: A noninvasive device for observing localized muscular fatigue. IEEE Trans Biomed Eng 29:760-768, 1982.

16. West JE, Busch-Vishniac IJ, Harshfield GA, Pickering GA: Foil electret transducer for blood pressure monitoring. $J$ Acoust Soc Am 74:680-683, 1983.

17. Wollaston WH: On the duration of muscle action. Philos Trans $R$ Soc $1-5,1810$ 